\title{
Appraisal of dispersion damping functions for the effective fragment potential method
}

\author{
Kevin Carter-Fenk* and John M. Herbert ${ }^{\dagger}$ \\ Department of Chemistry and Biochemistry, The Ohio State University, Columbus, OH 43210
}

(Dated: January 24, 2022)

\begin{abstract}
The effective fragment potential (EFP) is a polarizable force field whose physically-motivated functional form is parameterized in an automated way from ab initio calculations, and whose dispersion potential has been suggested as a correction for Hartree-Fock or density functional theory calculations. However, the parameter-free dispersion damping potentials that are currently used in EFP do not follow from a rigorous derivation and do not satisfy simple limits for the dispersion energy. We introduce several new damping expressions that correct these deficiencies, then evaluate their performance alongside existing damping functions using a new database of ionic liquid constituents. This data set, which we call IL195 $\times 8$, consists of complete-basis coupled-cluster interaction energies for 195 ion pairs at each of 8 different intermolecular separations. Ultimately, we recommend a new parameter-free dispersion damping function as a replacement for the one that is currently used in EFP.
\end{abstract}

\section{Introduction}

The effective fragment potential (EFP) method is a widely used polarizable force field that is obtained by automated parameterization of a physicallymotivated functional form, based on ab initio quantum chemistry. ${ }^{1-4}$ It has been used in hybrid quantum mechanics/molecular mechanics (QM/MM) calculations, both in the ground state $^{5-11}$ and for vertical excitation spectra. ${ }^{12-16}$ Motivated by the success of empirical dispersion potentials in density functional theory (DFT), ${ }^{17,18}$ another potential application of EFP is to use its dispersion component as a parameter-free correction for DFT or even Hartree-Fock (HF) theory. ${ }^{19,20}$ The $\mathrm{HF}$ case is interesting insofar as dispersion-corrected HF theory does not involve any double-counting of electron correlation effects in the "middle-range" region, corresponding to non-bonded close-contact distances in van der Waals complexes, which is a concern in dispersioncorrected DFT. ${ }^{17,21-23}$ Empirical dispersion corrections have also been introduced in the context of second-order Møller-Plesset perturbation theory (MP2), ${ }^{24,25}$ because while MP2 does provide a first-principles description of dispersion, it is not a quantitative method for that purpose. ${ }^{25-32}$ Dispersion corrections have also been introduced for the closely-related method of symmetryadapted perturbation theory (SAPT). ${ }^{31-40}$ It is in the context that we had occasion to revisit EFP dispersion.

\section{Theory}

The dispersion component of the EFP energy is expressed in terms of $C_{6}$ coefficients that represent the leadingorder dipole-dipole contribution to dispersion. ${ }^{3}$ The $C_{6}$

\footnotetext{
*Present address: Dept. of Chemistry, University of California, Berkeley CA, USA

therbert@chemistry.ohio-state.edu
}

coefficients are obtained from the well-known expression involving frequency-dependent, isotropic atomic polarizabilities $\left(\alpha_{p}\right)$ evaluated at imaginary frequencies. ${ }^{41-45}$ That expression, which is usually attributed to Casimir and Polder, ${ }^{46}$ is given by ${ }^{44,47-51}$

$$
C_{6, p q}=\frac{3 \hbar}{\pi} \int_{0}^{\infty} \alpha_{p}(\mathrm{i} \omega) \alpha_{q}(\mathrm{i} \omega) d \omega .
$$

The total dispersion energy is

$$
E_{\mathrm{disp}}=-\sum_{p \in A} \sum_{q \in B} \frac{C_{6, p q}}{R_{p q}^{6}} .
$$

In EFP, the centers $p$ and $q$ are not atoms but rather centroids of localized molecular orbital (LMOs), whose spherically-averaged polarizabilities $\alpha_{p}$ are used in Eq. (1). Like all other dispersion potentials of the $-C_{6} / R^{6}$ form, Eq. (2) exhibits a singularity as $R \rightarrow 0$ and a damping function is required to avoid divergence at short range.

In this work, we focus on the "parameter-free" version of EFP, sometimes called EFP2 to distinguish it from an earlier model that involved some fitting. ${ }^{4}$ The dispersion damping function that is used in this version of EFP is intended to be a modification of the Tang-Toennies damping function, ${ }^{52}$

$$
f_{n}\left(R_{p q}\right)=1-\exp \left(-b R_{p q}\right) \sum_{k=0}^{n} \frac{\left(b R_{p q}\right)^{k}}{k !} .
$$

The order $n$ is taken to match $R_{p q}^{-n}$ in the dispersion expression, meaning $n=6$ for use with Eq. (2). The exponential parameter $b$ is often written in the form

$$
b=a_{1} R_{p q}^{\mathrm{vdW}}+a_{2}
$$

where $a_{1}$ and $a_{2}$ are additional parameters and $R_{p q}^{\mathrm{vdW}}$ is an effective van der Waals (vdW) contact distance between centers $p$ and $q$. Because EFP uses spherical Gaussian LMOs, Slipchenko and Gordon (SG) introduced a 
Gaussian analogue of Eq. (3), ${ }^{53}$ namely

$$
f_{n}\left(R_{p q}\right)=1-\exp \left(-\zeta R_{p q}^{2}\right) \sum_{k=0}^{n / 2} \frac{\left(\zeta R_{p q}^{2}\right)^{k}}{k !} .
$$

As compared to the summation in Eq. (3), this modified form contains only even powers of $R_{p q}$ but it terminates at the same order, $R_{p q}^{n}$. This complements the denominator in the dispersion expression to all even powers $R_{p q}^{-2 k}$.

Noting that the overlap integral for two spherical Gaussian functions with exponents $\zeta$ and center-to-center distance $R_{p q}$ is

$$
S_{p q}=\exp \left(-\zeta R_{p q}^{2} / 2\right),
$$

SG eliminate the parameter $\zeta$ in Eq. (5) by substituting the exponent that is obtained during localization of the canonical MOs. The resulting expression is written in terms of the overlaps $S_{p q}$ between LMOs, rather than the distance $R_{p q}$ per se: ${ }^{53}$

$$
f_{n}^{\text {orig }}\left(S_{p q}\right)=1-S_{p q}^{2} \sum_{k=0}^{n / 2} \frac{\left(-2 \ln \left|S_{p q}\right|\right)^{k}}{k !} .
$$

This eliminates any fitting parameters beyond the Gaussian exponents that are taken from standard Gaussian basis sets. In Ref. 53, however, the summation in Eq. (7) is truncated at order $(n-2) / 2$ for $n=6$, resulting in

$$
f_{6}^{\text {orig }}\left(S_{p q}\right)=1-S_{p q}^{2}\left[1-2 \ln \left|S_{p q}\right|+2\left(\ln \left|S_{p q}\right|\right)^{2}\right] .
$$

This is the form that the functions takes in the libEFP library, ${ }^{54}$ and thus defines what we will call the "original" version of the parameter-free EFP dispersion damping function.

In order to be consistent with the TT damping function, or even its Gaussian modification in Eq. (5), the damping function in Eq. (8) ought to contain one additional term. That way, the damping expression meets the dispersion potential with a commensurate power of $R_{p q}^{n}$. Adding the missing term to Eq. (8) affords

$f_{6}\left(S_{p q}\right)=1-S_{p q}^{2}\left[1-2 \ln \left|S_{p q}\right|+2\left(\ln \left|S_{p q}\right|\right)^{2}-\frac{4}{3}\left(\ln \left|S_{p q}\right|\right)^{3}\right]$,

where the four terms in square brackets are intended to scale like $R_{p q}^{0}, R_{p q}^{2}, R_{p q}^{4}$, and $R_{p q}^{6}$, respectively, as in Eq. (5) with $n=6$. Instead, the expression in Eq. (8) has been applied to the $C_{7}$ component of EFP dispersion, ${ }^{55}$ despite the absence of any term in the numerator with a distance dependence greater than $R_{p q}^{4}$.

Furthermore, while the ansatz in Eq. (6) does eliminate parameters from the damping function, it also makes the tacit assumption that all pairs of LMOs have the same Gaussian exponent, $\zeta$. If centers $p$ and $q$ have different exponents $\alpha$ and $\beta$, then a more appropriate expression is

$$
S_{p q}=\exp \left(-\frac{\alpha \beta}{\alpha+\beta} R_{p q}^{2}\right)
$$

Setting $\gamma=\alpha \beta /(\alpha+\beta)$ and using

$$
S_{p q}=\exp \left(-\gamma R_{p q}^{2}\right)
$$

as the damping function in Eq. (5), one obtains a slightly different form for the damping function:

$$
f_{n}^{\mathrm{rev}}\left(S_{p q}\right)=1-S_{p q} \sum_{k=0}^{n / 2} \frac{\left(-\ln \left|S_{p q}\right|\right)^{k}}{k !} .
$$

For $n=6$ this is

$f_{6}^{\mathrm{rev}}\left(S_{p q}\right)=1-S_{p q}\left[1-\ln \left|S_{p q}\right|+\frac{1}{2}\left(\ln \left|S_{p q}\right|\right)^{2}-\frac{1}{6}\left(\ln \left|S_{p q}\right|\right)^{3}\right]$,

and we call this the "revised" parameter-free dispersion damping function.

The original parameter-free damping function in Eq. (8) was introduced in $2009,{ }^{53}$ but has been updated in more recent work to incorporate odd powers of $R$. This is intended to provide a more consistent expression once a $C_{7}$ term is introduced in the EFP dispersion energy. The updated damping function is ${ }^{19,20}$

$$
f_{n}^{\text {gen }}\left(S_{p q}\right)=1-S_{p q}^{2} \sum_{k=0}^{n} \frac{\left(-2 \ln \left|S_{p q}\right|\right)^{k / 2}}{k !},
$$

which we will call a "generalized" EFP dispersion damping function. However, the erroneous assumption of a single Gaussian exponent, leading to Eq. (7) in the original formulation, is repeated in this more recent expression. As such, we also introduce a "revised general" damping function of the form

$$
f_{n}^{\text {rev-gen }}\left(S_{p q}\right)=1-S_{p q} \sum_{k=0}^{n} \frac{\left(-\ln \left|S_{p q}\right|\right)^{k / 2}}{k !},
$$

where we have not made any assumptions about the nature of the Gaussian exponents. It is worth noting that neither Eq. (14) nor Eq. (15) is bounded on the interval $[0,1]$, which is undesirable from a rigorous standpoint. This has likely gone unnoticed in practice because divergences occur only very close to $R_{p q}=0$ and in most real systems the steric repulsion will preclude access to these problematic geometries.

Finally, we introduce two novel damping functions to be investigated alongside those discussed above. First, we write an exact Tang-Toennies expression in terms of the overlap, which puts the generalized formulas presented above on a more rigorous foundation:

$$
f_{n}^{\mathrm{TT}}\left(R_{p q}\right)=1-e^{-\left(\gamma R_{p q}^{2}\right)^{1 / 2}} \sum_{k=0}^{n} \frac{\left(\gamma R_{p q}^{2}\right)^{k / 2}}{k !} .
$$

Using the overlap expression in Eq. (11), one may eliminate the parameter

$$
\gamma=\frac{-\ln \left|S_{p q}\right|}{R_{p q}^{2}}
$$


Table 1: Definitions of various damping functions for EFP dispersion. ${ }^{a}$

\begin{tabular}{lcc}
\hline \hline Damping Function & Functional Form & $\lim _{R \rightarrow 0}\left(-f_{6} / R^{6}\right)$ \\
\hline Original $^{b}$ & $1-S_{p q}^{2} \sum_{k=0}^{(n-2) / 2} \frac{1}{k !}\left(-2 \ln \left|S_{p q}\right|\right)^{k}$ & $-4 \gamma / 3$ \\
Generalized $^{c}$ & $1-S_{p q}^{2} \sum_{k=0}^{n} \frac{1}{k !}\left(-2 \ln \left|S_{p q}\right|\right)^{k / 2}$ & $\infty$ \\
Revised $^{n}$ & $1-S_{p q} \sum_{k=0}^{n / 2} \frac{1}{k !}\left(-\ln \left|S_{p q}\right|\right)^{k}$ & 0 \\
Revised General & $1-S_{p q} \sum_{k=0}^{n} \frac{1}{k !}\left(-\ln \left|S_{p q}\right|\right)^{k / 2}$ & $\infty$ \\
Tang-Toennies & $1-e^{-\left(-\ln \left|S_{p q}\right|\right)^{1 / 2}} \sum_{k=0}^{n} \frac{1}{k !}\left(-\ln \left|S_{p q}\right|\right)^{k / 2}$ & 0 \\
Becke-Johnson & $R_{p q}^{n}\left[R_{p q}^{n}+\left(-R_{p q}^{2} / \ln \left|S_{p q}\right|\right)^{n / 2}\right]^{-1}$ & $-\gamma^{3}$ \\
\hline \hline
\end{tabular}

${ }^{a}$ Definitions are from this work unless otherwise noted, with $\gamma$ defined in Eq. (17).

${ }^{b}$ From Ref. 53.

${ }^{c}$ From Ref. 19.

and thus express the damping function in Eq. (16) in the equivalent form

$$
f_{n}^{\mathrm{TT}}\left(S_{p q}\right)=1-e^{-\left(-\ln \left|S_{p q}\right|\right)^{1 / 2}} \sum_{k=0}^{n} \frac{\left(-\ln \left|S_{p q}\right|\right)^{k / 2}}{k !} .
$$

Although this expression is bounded on $[0,1]$, it is likely to overdamp the interaction as a consequence of the exponential (rather than Gaussian) dependence on distance.

In order to avoid overdamping, we will also test a softer damping function based on the Becke-Johnson (BJ) form: ${ }^{56-59}$

$$
f_{n}^{\mathrm{BJ}}\left(R_{p q}\right)=\frac{R_{p q}^{n}}{R_{p q}^{n}+\left(a_{1} R_{p q}^{\mathrm{vdW}}+a_{2}\right)^{n}} .
$$

Recall that in modifying Eq. (3) for use with EFP, Gaussian exponents were taken as stand-ins for the TangToennies damping parameter $b$ in Eq. (4). We apply the same substitution here, only using the general form of the Gaussian overlap exponents, $\gamma=\alpha \beta /(\alpha+\beta)$. Note that $\gamma$ in Eq. (17) has dimensions of (length) ${ }^{-2}$. Manipulating that expression to substitute a $\gamma$-dependent factor in place of $b^{n}$ in the denominator of Eq. (19) affords the expression

$$
f_{n}^{\mathrm{BJ}}\left(R_{p q}, S_{p q}\right)=\frac{R_{p q}^{n}}{R_{p q}^{n}+\left(-R_{p q}^{2} / \ln \left|S_{p q}\right|\right)^{n / 2}} .
$$

This overlap-based BJ damping function retains the parameter-free philosophy of EFP but is trivially extensible to odd powers of $R$ without resorting to augmentations that render the function unbound on the interval $[0,1]$.

Table 1 summarizes the damping functions introduced above. A successful damping function must prevent $E_{\text {disp }}$ from diverging as $R \rightarrow 0$, regardless of the fine details of how it performs otherwise. This limit provides a useful first assessment of the validity of any damping function, and Table 1 reports the the limiting value $\lim _{R \rightarrow 0}\left(-f_{6} / R^{6}\right)$ for each damping function. The original EFP damping function yields a finite, negative value for this limit. As such, while this function does successfully prevent divergence of the dispersion energy, it is not truly a Gaussian analogue of the TT damping function because the latter damps the dispersion energy to zero at $R=0 .{ }^{52}$ Failure to satisfy this limit is not fatal to the use of damped EFP dispersion, but it does exemplify the errors associated with early truncation of the original TangToennies expression. The revision suggested in Eq. (12) restores the proper limit, namely, $\lim _{R \rightarrow 0}\left(-f_{6} / R^{6}\right)=0$.

The generalized EFP dispersion damping expression [Eq. (14)], introduced to accommodate $C_{7}$ dispersion, ${ }^{19,20}$ does not prevent a singularity as $R \rightarrow 0$ and the revision introduced in Eq. (15) does not rescue it from this fate. In order to turn the generalized expression into a formally valid damping function, we abandon the concept of Gaussian damping altogether and return to the exponential Tang-Toennies expression in Eq. (16), as it restores the proper $R \rightarrow 0$ limit. Finally, the parameter-free BJ damping function in Eq. (20) exhibits a small but finite limit as $R \rightarrow 0$.

\section{Computational Methods}

For a numerical assessment of these various damping expression we turn to the HF plus dispersion method, which we denote as $\mathrm{HF}+\mathrm{D}(\mathrm{EFP})$ to indicate that the dispersion correction is obtained from EFP. Various forms of the $+\mathrm{D}(\mathrm{EFP})$ correction are considered, using the same form for the dispersion energy [Eq. (2)] but with various damping functions.

As an example of a "real-world" application where the $\mathrm{HF}+\mathrm{D}$ approach might be preferred to $\mathrm{DFT}+\mathrm{D}$, we consider a data set of ion pairs corresponding to common constituents of room-temperature ionic liquids. For such systems, Grimme et al. ${ }^{60}$ report that DFT calculations sometimes face convergence issues related to delocaliza- 
(a) equilibrium geometries

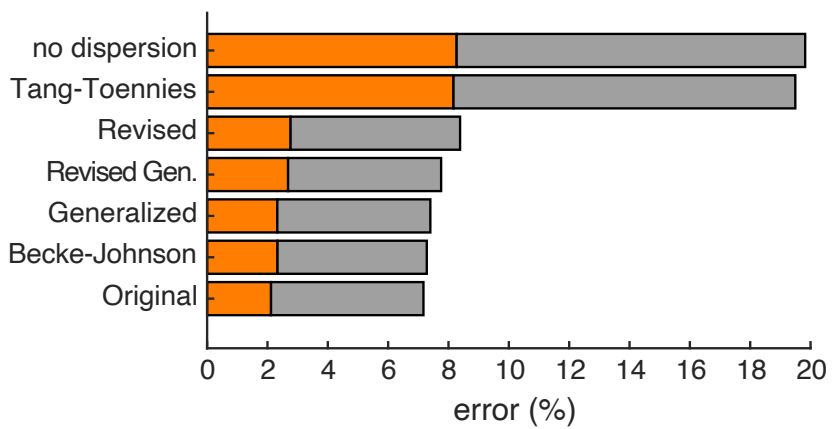

(b) full IL195×8 database

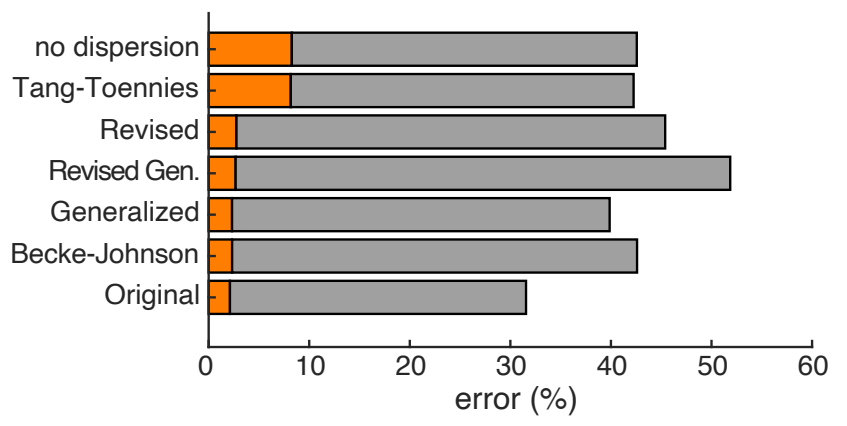

Figure 1: $\quad$ Error statistics in $\mathrm{HF}+\mathrm{D}(\mathrm{EFP}) / 6-311++\mathrm{G}(\mathrm{d}, \mathrm{p})$ interaction energies for the IL195 $\times 8$ data set, considering: (a) equilibrium geometries only, versus (b) the entire data set, including intermolecular separations ranging from $0.9 R_{e}$ to $2.0 R_{e}$ for each ion pair. Orange bars are mean absolute percentage errors and gray bars are maximum percentage errors, as compared to CCSD(T)/CBS benchmarks. The "no dispersion" method is conventional HF theory whereas other entries are HF $+\mathrm{D}(\mathrm{EFP})$ with various damping functions.

tion error and unphysical charge transfer, at least when semilocal functionals are employed. The $\mathrm{HF}+\mathrm{D}(\mathrm{EFP})$ method provides a potentially useful alternative in such cases, and indeed could be used as a general way to probe whether delocalization error is problematic in a particular case, without sacrificing the quantitatively important dispersion interaction. ${ }^{61,62}$

The data set assembled for this work is a subset of the IL-2013 database ${ }^{63}$ and consists of 195 unique ion pairs. We shift each dimer along the vector connecting the cation and anion centers of mass, in order to generate eight different geometries for each dimer corresponding to intermolecular separations ranging from $0.9 R_{e}$ to $2.0 R_{e}$, where $R_{e}$ is the equilibrium separation from the IL-2013 database. We call this data set IL195×8, and it consists of 1,560 individual structures whose interaction energies we have computed at the level of coupled-cluster theory with single, double, and perturbative triple excitations $[\operatorname{CCSD}(\mathrm{T})]$, using the domain-localized pair natural orbital (DLPNO) implementation of $\operatorname{CCSD}(\mathrm{T})^{64,65}$ in the ORCA program. ${ }^{66}$ Tight cutoffs are used because the accuracy of the DLPNO approximation is known to be quite sensitive to these thresholds for noncovalent interaction energies. ${ }^{67,68}$ DLPNO-CCSD(T) interaction energies were extrapolated to the complete basis-set (CBS) limit using a two-point formula ${ }^{69}$ with aug-cc-pVTZ and aug-cc-pVQZ. Additional details are provided in the Supplementary Material.

EFP parameters for each cation and each anion in the data set were computed at the HF/6-311++G(3df,2p) level, using the GAMESS program. ${ }^{70}$ We use the same (rigid) monomer geometries and thus the same EFP parameters for each of the eight intermolecular separations. The EFP energy calculations discussed below were performed at the $\mathrm{HF}+\mathrm{D}(\mathrm{EFP}) / 6-311++\mathrm{G}(\mathrm{d}, \mathrm{p})$ level using a locally-modified version of the Q-Chem program, ${ }^{71}$ which includes an interface ${ }^{72}$ to the libEFP library. ${ }^{54}$

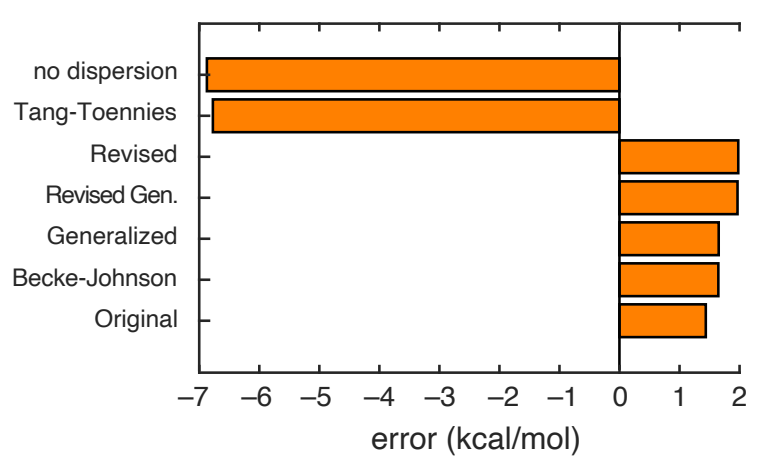

Figure 2: $\quad$ Mean errors in $\mathrm{HF}+\mathrm{D}(\mathrm{EFP}) / 6-311++\mathrm{G}(\mathrm{d}, \mathrm{p})$ interaction energies for the IL195 $\times 8$ data set using various damping functions for EFP dispersion. Positive errors reflect systematic overbinding and negative errors indicate underbinding.

\section{Results and Discussion}

Mean absolute percentage errors for the IL195 $\times 8$ data set, comparing $\mathrm{HF}+\mathrm{D}(\mathrm{EFP}) / 6-311++\mathrm{G}(\mathrm{d}, \mathrm{p})$ calculations to CCSD(T)/CBS benchmarks, are shown in Fig. 1. With the notable exception of the Tang-Toennies damping function, each of the various damping procedures performs similarly with errors of about $2 \%$. The TangToennies function, however, damps $E_{\text {disp }}$ to such an extent that the dispersion-corrected method is no better than plain HF theory without any dispersion correction at all! In the absence of a dispersion correction, or when the Tang-Toennies damping function is used, HF theory underbind the IL195 $\times 8$ complexes by an average of almost $7 \mathrm{kcal} / \mathrm{mol}$, as shown in Fig. 2. In fact, this underbinding was the original motivation for replacing the exponential in the Tang-Toennies damping function with a Gaussian function. ${ }^{53}$ 


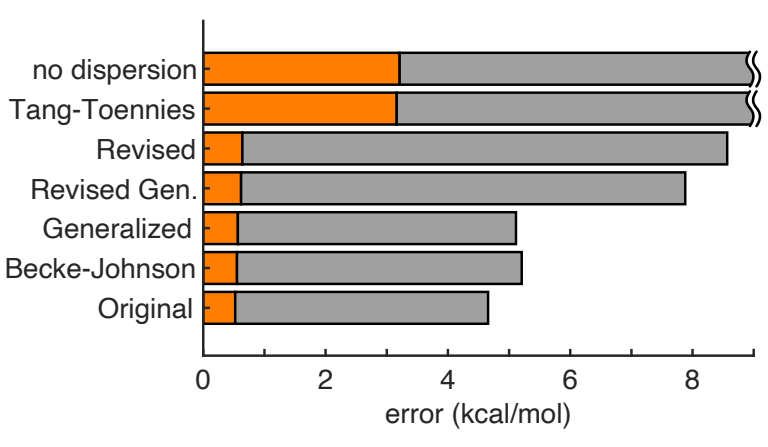

Figure 3: Error statistics in $\mathrm{HF}+\mathrm{D}(\mathrm{EFP}) /$ def2-TZVPPD interaction energies for the $\mathrm{S} 66 \times 8$ data set (with acetylene complexes removed) as compared to $\mathrm{CCSD}(\mathrm{T}) / \mathrm{CBS}$ benchmarks. Orange bars indicate the mean absolute errors and gray bars are maximum errors. The "no dispersion" method is conventional HF theory whereas other entries are $\mathrm{HF}+\mathrm{D}(\mathrm{EFP})$ with various damping functions. In two cases, the maximum error exceeds $8.5 \mathrm{kcal} / \mathrm{mol}$ and is cut off in this graph.

Setting aside the case of the Tang-Toennies damping function, the overall accuracy of the various damping functions is similar when measured in percentage terms. However, maximum errors in Fig. 1(b) highlight subtle nuances in the behavior of each damping potential as $R \rightarrow 0$ and each of the other damping functions systematically overbinds these dimers by $1-2 \mathrm{kcal} / \mathrm{mol}$; see Fig. 2. This is consistent with results indicating that EFP consistently overestimates dispersion interactions in ionic liquid constituent dimers, relative to third-order SAPT calculations. ${ }^{73}$ The original and generalized damping equations exhibit slightly smaller errors than their revised counterparts, although this indicates some error cancellation since only the revisions have a rigorous theoretical foundation. The BJ damping function affords results comparable to those of the original EFP damping function but without ad hoc approximations such as missing terms. We recommend this version for general use with EFP dispersion.

We have also assessed the performance of $\mathrm{HF}+\mathrm{D}(\mathrm{EFP})$ on the $\mathrm{S} 66 \times 8$ data set of dimers, ${ }^{74}$ where the monomers are all charge-neutral, in order to test whether the results above are somehow an artifact of the ionic liquid data set. (Dimers containing acetylene were excluded from these calculations because the Q-Chem/libEFP interface does not handle linear fragments at the present time.) Error statistics are shown in Fig. 3 and suggest that the general behavior is quite similar to what we observed for ionic liquid constituent dimers.

\section{Conclusions}

We have examined various flavors of $\mathrm{HF}+\mathrm{D}(\mathrm{EFP})$ using two sizable data sets of noncovalent dimers. Overall, we find that the damping functions currently used for EFP dispersion are unsatisfactory from a formal standpoint as the power series in the modified Tang-Toennies damping function is truncated too early. Furthermore, replacement of an empirical damping parameter with a spherical Gaussian overlap integral fails to account for asymmetric Gaussian exponents on different centers. These errors lead to damping functions that do not satisfy correct limiting conditions. In particular, a generalized damping function that was introduced to extend the original formulation to odd powers of $R$ (in order to incorporate $R^{-7}$ dispersion) is divergent as $R \rightarrow 0$ and thus does not serve as a damping function at all when the intermolecular separation is small.

Ultimately, we recommend the parameter-free, BJstyle dispersion damping function introduced in Eq. (20) as a replacement for the modified Tang-Toennies function that is presently employed in EFP. For the data sets examined here, the $\mathrm{HF}+\mathrm{D}(\mathrm{EFP})$ method in conjunction with new damping function exhibits an accuracy that rivals existing EFP damping functions, yet the new approach is based on a correct theoretical framework and is extensible to odd powers of $R$. Given the extensive use of EFP across myriad computational chemistry applications, we expect that following this recommendation will minimize errors in many future studies by putting EFP dispersion on a more rigorous footing.

\section{Acknowledgments}

The authors warmly acknowledge Prof. Peter Gill as the founder of the Q-Chem project, which he has successfully nurtured over more than a quarter of a century and which has played a major role in the authors' professional lives. This work was supported by the U.S. Department of Energy, Office of Basic Energy Sciences, Division of Chemical Sciences, Geosciences, and Biosciences under Award No. DE-SC0008550. K.C.-F. acknowledges a Presidential Fellowship from The Ohio State University. Calculations were run at the Ohio Supercomputer Center under project no. PAA-0003. ${ }^{75}$ J.M.H. serves on the board of directors of Q-Chem, Inc.

\section{Supplementary Material Available}

IL195×8 geometries and CCSD(T)/CBS energies. 
1 P. N. Day, J. H. Jensen, M. S. Gordon, S. P. Webb, W. J. Stevens, M. Krauss, D. Garmer, H. Basch, and D. Cohen, "An effective fragment method for modeling solvent effects in quantum mechanical calculations", J. Chem. Phys., 105, 1968-1986 (1996).

2 M. S. Gordon, M. A. Freitag, P. Bandyopadhyay, J. H. Jensen, V. Kairys, and W. J. Stevens, "The effective fragment potential method: A QM-based MM approach to modeling environmental effects in chemistry", J. Phys. Chem. A, 105, 293-307 (2001).

3 D. Ghosh, D. Kosenkov, V. Vanovschi, C. F. Williams, J. M. Herbert, M. S. Gordon, M. W. Schmidt, L. V. Slipchenko, and A. I. Krylov, "Noncovalent interactions in extended systems described by the effective fragment potential method: Theory and application to nucleobase oligomers", J. Phys. Chem. A, 114, 12739-12754 (2010).

${ }^{4}$ L. V. Slipchenko and P. K. Gurunathan, "Effective fragment potential method: Past, present, and future", in Fragmentation: Toward Accurate Calculations on Complex Molecular Systems, M. S. Gordon, Ed.; Wiley: Hoboken, 2017; chapter 6, pages 183-208.

5 D. Ghosh, O. Isayev, L. V. Slipchenko, and A. I. Krylov, "Effect of solvation on the vertical ionization energy of thymine: From microhydration to bulk", J. Phys. Chem. A, 115, 6028-6038 (2011).

${ }^{6}$ D. Ghosh, A. Roy, R. Seidel, B. Winter, S. Bradforth, and A. I. Krylov, "First-principle protocol for calculating ionization energies and redox potentials of solvated molecules and ions: Theory and application to aqueous phenol and phenolate", J. Phys. Chem. B, 116, 7269-7280 (2012).

7 R. Chakraborty, S. Bose, and D. Ghosh, "Effect of solvation on the ionization of guanine nucleotide: A hybrid QM/ EFP study", J. Comput. Chem., 38, 2528-2537 (2017).

8 S. Bose and D. Ghosh, "An interaction energy driven biased sampling technique: A faster route to ionization spectra in condensed phase", J. Comput. Chem., 38, 2248-2257 (2017).

9 R. N. Tazhigulov, P. K. Gurunathan, Y. Kim, L. V. Slipchenko, and K. B. Bravaya, "Polarizable embedding for simulating redox potentials of biomolecules", Phys. Chem. Chem. Phys., 21, 11642-11650 (2019).

10 T. Sattasathuchana, P. Xu, and M. S. Gordon, "An accurate quantum-based approach to explicit solvent effects: Interfacing the general effective fragment potential method with ab initio electronic structure theory", J. Phys. Chem. A, 123, 8460-8475 (2019).

11 K.-Y. Liu and J. M. Herbert, "Energy-screened many-body expansion: A practical yet accurate fragmentation method for quantum chemistry", J. Chem. Theory Comput., 16, 475-487 (2020).

12 L. V. Slipchenko, "Solvation of the excited states of chromophores in polarizable environment: Orbital relaxation versus polarization", J. Phys. Chem. A, 114, 8824-8830 (2010).

13 P. Arora, L. V. Slipchenko, S. P. Webb, A. DeFusco, and M. S. Gordon, "Solvent-induced frequency shifts: Configuration interaction singles combined with the effective fragment potential method", J. Phys. Chem. A, 114, 67426750 (2010).

14 A. DeFusco, N. Minezawa, L. V. Slipchenko, F. Zahariev, and M. S. Gordon, "Modeling solvent effects on electronic excited states", J. Phys. Chem. Lett., 2, 2184-2192 (2011).

15 D. Ghosh, "Perturbative approximation to hybrid equation of motion coupled cluster/effective fragment potential method", J. Chem. Phys., 140, 094101:1-9 (2014).

16 D. Ghosh, "Hybrid equation-of-motion coupled-cluster/ effective fragment potential method: A route toward understanding photoprocesses in the condensed phase", J. Phys. Chem. A, 121, 741-752 (2017).

17 S. Grimme, "Density functional theory with London dispersion corrections", WIREs Comput. Mol. Sci., 1, 211228 (2011).

18 S. Grimme, A. Hansen, J. G. Brandenburg, and C. Bannwarth, "Dispersion-corrected mean-field electronic structure methods", Chem. Rev., 116, 5105-5154 (2016).

19 E. B. Guidez and M. S. Gordon, "Dispersion correction derived from first principles for density functional theory and Hartree-Fock theory", J. Phys. Chem. A, 119, 21612168 (2015).

20 S. Kim, C. M. Kaliszewski, E. B. Guidez, and M. S. Gordon, "Benchmarking the effective fragment potential dispersion correction on the S22 test set", J. Phys. Chem. A, 122, 4076-4084 (2018).

21 M. Shahbaz and K. Szalewicz, "Do semilocal densityfunctional approximations recover dispersion energies at small intermonomer separations?", Phys. Rev. Lett., 121, 113402:1-6 (2018).

22 M. Shahbaz and K. Szalewicz, "Evaluation of methods for obtaining dispersion energies used in density functional calculations of intermolecular interactions", Theor. Chem. Acc., 138, 25:1-17 (2019).

23 A. J. A. Price, K. R. Bryenton, and E. R. Johnson, "Requirements for an accurate dispersion-corrected density functional", J. Chem. Phys., 154, 230902:1-12 (2021).

24 C. Greenwell, J. Řezáč, and G. J. O. Beran, Spincomponent-scaled and dispersion-corrected second-order Møller-Plesset perturbation theory: A path toward chemical accuracy. (DOI: 10.33774/chemrxiv-2021-jdv3c.)

25 J. Řezác, C. Greenwell, and G. J. O. Beran, "Accurate noncovalent interactions via dispersion-corrected secondorder Møller-Plesset perturbation theory", J. Chem. Theory Comput., 14, 4711-4721 (2018).

${ }^{26}$ M. O. Sinnokrot and C. D. Sherrill, "Highly accurate coupled cluster potential energy curves for the benzene dimer: Sandwich, T-shaped, and parallel-displaced configurations", J. Phys. Chem. A, 108, 10200-10207 (2004).

27 A. Heßelmann, "Improved supermolecular second order Møller-Plesset intermolecular interaction energies using time-dependent density functional response theory", J. Chem. Phys., 128, 144112:1-9 (2008).

28 J. M. Herbert, L. D. Jacobson, K. U. Lao, and M. A. Rohrdanz, "Rapid computation of intermolecular interactions in molecular and ionic clusters: Self-consistent polarization plus symmetry-adapted perturbation theory", Phys. Chem. Chem. Phys., 14, 7679-7699 (2012).

${ }^{29}$ K. U. Lao and J. M. Herbert, "Symmetry-adapted perturbation theory with Kohn-Sham orbitals using nonempirically tuned, long-range-corrected density functionals", J. Chem. Phys., 140, 044108:1-8 (2014).

30 B. Nguyen, G. P. Chen, M. M. Agee, A. M. Burow, M. Tang, and F. Furche, "Divergence of many-body perturbation theory for noncovalent interactions of large 
molecules", J. Chem. Theory Comput., 16, 2258-2273 (2020).

${ }^{31}$ K. U. Lao and J. M. Herbert, "Atomic orbital implementation of extended symmetry-adapted perturbation theory (XSAPT) and benchmark calculations for large supramolecular complexes", J. Chem. Theory Comput., 14, 2955-2978 (2018).

${ }^{32}$ K. Carter-Fenk, K. U. Lao, and J. M. Herbert, "Predicting and understanding non-covalent interactions using novel forms of symmetry-adapted perturbation theory", Acc. Chem. Res., 54, 3679-3690 (2021).

33 A. Heßelmann, "Comparison of intermolecular interaction energies from SAPT and DFT including empirical dispersion contributions", J. Phys. Chem. A, 115, 11321-11330 (2011).

${ }^{34}$ K. U. Lao and J. M. Herbert, "Accurate intermolecular interactions at dramatically reduced cost: XPol+SAPT with empirical dispersion", J. Phys. Chem. Lett., 3, 3241-3248 (2012).

35 L. D. Jacobson, R. M. Richard, K. U. Lao, and J. M. Herbert, "Efficient monomer-based quantum chemistry methods for molecular and ionic clusters", Annu. Rep. Comput. Chem., 9, 25-58 (2013).

${ }^{36}$ K. U. Lao and J. M. Herbert, "An improved treatment of empirical dispersion and a many-body energy decomposition scheme for the explicit polarization plus symmetryadapted perturbation theory (XSAPT) method", J. Chem. Phys., 139, 034107:1-16 (2013). Erratum: J. Chem. Phys. 140, 119901 (2014).

37 K. U. Lao and J. M. Herbert, "Accurate and efficient quantum chemistry calculations of noncovalent interactions in many-body systems: The XSAPT family of methods", J. Phys. Chem. A, 119, 235-253 (2015).

${ }^{38}$ K. Carter-Fenk, K. U. Lao, K.-Y. Liu, and J. M. Herbert, "Accurate and efficient $a b$ initio calculations for supramolecular complexes: Symmetry-adapted perturbation theory with many-body dispersion", J. Phys. Chem. Lett., 10, 2706-2714 (2019).

39 K.-Y. Liu, K. Carter-Fenk, and J. M. Herbert, "Selfconsistent charge embedding at very low cost, with application to symmetry-adapted perturbation theory", J. Chem. Phys., 151, 031102:1-7 (2019).

40 J. B. Schriber, D. A. Sirianni, D. G. A. Smith, L. A. Burns, D. Sitkoff, D. L. Cheney, and C. D. Sherrill, "Optimized damping parameters for empirical dispersion corrections to symmetry-adapted perturbation theory", J. Chem. Phys., 154, 234107:1-10 (2021).

41 A. D. McLachlan, "Retarded dispersion forces between molecules", Proc. R. Soc. Lond. A, 271, 387-401 (1963).

42 A. D. McLachlan, "Effect of the medium on dispersion forces in liquids", Discuss. Faraday Soc., 40, 239-245 (1965).

43 H. C. Longuet-Higgins, "Intermolecular forces", Discuss. Faraday Soc., 40, 7-18 (1965).

44 K. T. Tang, "Dynamic polarizabilities and van der Waals coefficients", Phys. Rev., 177, 108-114 (1969).

45 R. McWeeny, "Weak interactions between molecules", Croatica Chem. Acta, 57, 865-878 (1984).

${ }^{46}$ H. B. G. Casimir and B. Polder, "The influence of retardation on the London-van der Waals forces", Phys. Rev., 73, 360-372 (1948).

47 P. W. Langhoff and M. Karplus, "Application of Padé approximants to dispersion force and optical polarizability computations", in The Padé Approximant in Theoretical
Physics, G. A. Baker Jr. and J. L. Gammel, Eds., Vol. 71 of Mathematics in Science and Engineering; Academic Press: New York, 1970; chapter 2, pages 41-97.

48 A. Szabo and N. S. Ostlund, "The correlation energy in the random phase approximation: Intermolecular forces between closed-shell systems", J. Chem. Phys., 67, 43514360 (1977).

49 I. G. Kaplan, Intermolecular Interactions: Physical Picture, Computational Methods and Model Potentials, John Wiley \& Sons: Chichester, United Kingdom, 2006.

${ }^{50}$ V. A. Parsegian, Van der Waals Forces: A Handbook for Biologists, Chemists, Engineers, and Physicists, Cambridge University Press: New York, 2006.

51 J. M. Herbert, "Dielectric continuum methods for quantum chemistry", WIREs Comput. Mol. Sci., 11, e1519:173 (2021)

52 K. T. Tang and J. P. Toennies, "An improved simple model for the van der Waals potential based on universal damping functions for the dispersion coefficients", J. Chem. Phys., 80, 3726-3741 (1984).

${ }^{53}$ L. V. Slipchenko and M. S. Gordon, "Damping functions in the effective fragment potential", Mol. Phys., 107, 9991016 (2009).

54 I. A. Kaliman and L. V. Slipchenko, "LIBEFP: A new parallel implementation of the effective fragment potential method as a portable software library", J. Comput. Chem., 34, 2284-2292 (2013).

55 P. Xu, F. Zahariev, and M. S. Gordon, "The $R^{-7}$ dispersion interaction in the general effective fragment potential method", J. Chem. Theory Comput., 10, 1576-1587 (2014).

56 A. D. Becke and E. R. Johnson, "A density-functional model of the dispersion interaction", J. Chem. Phys., 123, 154101:1-9 (2005).

57 E. R. Johnson and A. D. Becke, "A post-Hartree-Fock model of intermolecular interactions", J. Chem. Phys., 123, 024101:1-7 (2005).

58 E. R. Johnson and A. D. Becke, "A post-Hartree-Fock model of intermolecular interactions: Inclusion of higherorder corrections", J. Chem. Phys., 124, 174104:1-9 (2006).

59 S. Grimme, S. Ehrlich, and L. Goerigk, "Effect of the damping function in dispersion corrected density functional theory", J. Comput. Chem., 32, 1456-1465 (2011).

60 S. Grimme, W. Hujo, and B. Kirchner, "Performance of dispersion-corrected density functional theory for the interactions in ionic liquids", Phys. Chem. Chem. Phys., 14, 4875-4883 (2012).

61 Z. C. Holden, B. Rana, and J. M. Herbert, "Analytic energy gradients for the QM/MM-Ewald method using atomic charges derived from the electrostatic potential: Theory, implementation, and application to ab initio molecular dynamics of the aqueous electron", J. Chem. Phys., 150, 144115:1-20 (2019).

62 S. Dasgupta, B. Rana, and J. M. Herbert, "Ab initio investigation of the resonance Raman spectrum of the hydrated electron", J. Phys. Chem. B, 123, 8074-8084 (2019).

63 S. Zahn, D. R. MacFarlane, and E. I. Izgorodina, "Assessment of Kohn-Sham density functional theory and MøllerPlesset perturbation theory for ionic liquids", Phys. Chem. Chem. Phys., 15, 13664-13675 (2013).

${ }^{64}$ C. Riplinger and F. Neese, "An efficient and near linear scaling pair natural orbital based local coupled cluster method", J. Chem. Phys., 138, 034106:1-18 (2013). 
65 C. Riplinger, B. Sandhoefer, A. Hansen, and F. Neese, "Natural triple excitations in local coupled cluster calculations with pair natural orbitals", J. Chem. Phys., 139, 134101:1-13 (2013).

66 F. Neese, "Software update: The ORCA program system, version 4.0", WIREs Comput. Mol. Sci., 8, e1327:1-6 (2017).

67 K. Sorathia and D. P. Tew, "Basis set extrapolation in pair natural orbital theories", J. Chem. Phys., 153, 174112:1-8 (2020).

68 F. Ballesteros, S. Dunivan, and K. U. Lao, "Coupled cluster benchmarks of large noncovalent complexes: The L7 dataset as well as DNA-ellipticine and buckycatcherfullerene", J. Chem. Phys., 154, 154104:1-12 (2021).

${ }^{69}$ F. Neese and E. F. Valeev, "Revisiting the atomic natural orbital approach for basis sets: Robust systematic basis sets for explicitly correlated and conventional correlated ab initio methods", J. Chem. Theory Comput., 7, 33-43 (2011).

70 G. M. J. Barca, C. Bertoni, L. Carrington, D. Datta, N. De Silva, J. E. Deustua, D. G. Fedorov, J. R. Gour, A. O. Gunina, E. Guidez, T. Harville, S. Irle, J. Ivanic, K. Kowalski, S. S. Leang, H. Li, W. Li, J. J. Lutz, I. Magoulas, J. Mato, V. Mironov, H. Nakata, B. Q. Pham, P. Piecuch, D. Poole, S. R. Pruitt, A. P. Rendell, L. B. Roskop, K. Ruedenberg, T. Sattasathuchana, M. W. Schmidt, J. S. L. Slipchenko, M. Sosokina, V. Sundriyal, A. Tiwari, J. L. G. Vallejo, B. Westheimer, M. Włoch, P. Xu, F. Zahariev, and M. S. Gordon, "Recent developments in the general atomic and molecular electronic structure system", J. Chem. Phys., 152, 154102:1-27 (2020).

${ }^{71}$ E. Epifanovsky, A. T. B. Gilbert, X. Feng, J. Lee, Y. Mao, N. Mardirossian, P. Pokhilko, A. F. White, M. P. Coons, A. L. Dempwolff, Z. Gan, D. Hait, P. R. Horn, L. D. Jacobson, I. Kaliman, J. Kussmann, A. W. Lange, K. U. Lao, D. S. Levine, J. Liu, S. C. McKenzie, A. F. Morrison, K. D. Nanda, F. Plasser, D. R. Rehn, M. L. Vidal, Z.-Q. You, Y. Zhu, B. Alam, B. J. Albrecht, A. Aldossary, E. Alguire, J. H. Andersen, V. Athavale, D. Barton, K. Begam, A. Behn, N. Bellonzi, Y. A. Bernard, E. J. Berquist, H. G. A. Burton, A. Carreras, K. CarterFenk, R. Chakraborty, A. D. Chien, K. D. Closser, V. Cofer-Shabica, S. Dasgupta, M. de Wergifosse, J. Deng, M. Diedenhofen, H. Do, S. Ehlert, P.-T. Fang, S. Fatehi, Q. Feng, T. Friedhoff, J. Gayvert, Q. Ge, G. Gidofalvi, M. Goldey, J. Gomes, C. E. González-Espinoza, S. Gulania, A. O. Gunina, M. W. D. Hanson-Heine, P. H. P. Harbach, A. Hauser, M. F. Herbst, M. Hernández Vera, M. Hodecker, Z. C. Holden, S. Houck, X. Huang, K. Hui, B. C. Huynh, M. Ivanov, A. Jász, H. Ji, H. Jiang, B. Kaduk, S. Kähler, K. Khistyaev, J. Kim, G. Kis, P. Klunzinger, Z. Koczor-Benda, J. H. Koh, D. Kosenkov,
L. Koulias, T. Kowalczyk, C. M. Krauter, K. Kue, A. Kunitsa, T. Kus, I. Ladjánszki, A. Landau, K. V. Lawler, D. Lefrancois, S. Lehtola, R. R. Li, Y.-P. Li, J. Liang, M. Liebenthal, H.-H. Lin, Y.-S. Lin, F. Liu, K.-Y. Liu, M. Loipersberger, A. Luenser, A. Manjanath, P. Manohar, E. Mansoor, S. F. Manzer, S.-P. Mao, A. V. Marenich, T. Markovich, S. Mason, S. A. Maurer, P. F. McLaughlin, M. F. S. J. Menger, J.-M. Mewes, S. A. Mewes, P. Morgante, J. W. Mullinax, K. J. Oosterbaan, G. Paran, A. C. Paul, S. K. Paul, F. Pavošević, Z. Pei, S. Prager, E. I. Proynov, A. Rák, E. Ramos-Cordoba, B. Rana, A. E. Rask, A. Rettig, R. M. Richard, F. Rob, E. Rossomme, T. Scheele, M. Scheurer, M. Schneider, N. Sergueev, S. M. Sharada, W. Skomorowski, D. W. Small, C. J. Stein, Y.C. Su, E. J. Sundstrom, Z. Tao, J. Thirman, G. J. Tornai, T. Tsuchimochi, N. M. Tubman, S. P. Veccham, O. Vydrov, J. Wenzel, J. Witte, A. Yamada, K. Yao, S. Yeganeh, S. R. Yost, A. Zech, I. Y. Zhang, X. Zhang, Y. Zhang, D. Zuev, A. Aspuru-Guzik, A. T. Bell, N. A. Besley, K. B. Bravaya, B. R. Brooks, D. Casanova, J.-D. Chai, S. Coriani, C. J. Cramer, G. Cserey, A. E. DePrince III, R. A. DiStasio Jr., A. Dreuw, B. D. Dunietz, T. R. Furlani, W. A. Goddard III, S. Hammes-Schiffer, T. Head-Gordon, W. J. Hehre, C.-P. Hsu, T.-C. Jagau, Y. Jung, A. Klamt, J. Kong, D. S. Lambrecht, W. Liang, N. J. Mayhall, C. W. McCurdy, J. B. Neaton, C. Ochsenfeld, J. A. Parkhill, R. Peverati, V. A. Rassolov, Y. Shao, L. V. Slipchenko, T. Stauch, R. P. Steele, J. E. Subotnik, A. J. W. Thom, A. Tkatchenko, D. G. Truhlar, T. Van Voorhis, T. A. Wesolowski, H. L. W. K. B. Whaley, P. M. Zimmerman, S. Faraji, P. M. W. Gill, M. Head-Gordon, J. M. Herbert, and A. I. Krylov, "Software for the frontiers of quantum chemistry: An overview of developments in the Q-Chem 5 package", J. Chem. Phys., 155, 084801:1-59 (2021).

72 D. Ghosh, D. Kosenkov, V. Vanovschi, J. Flick, I. Kaliman, Y. Shao, A. T. B. Gilbert, A. I. Krylov, and L. V. Slipchenko, "Effective fragment potential method in QCHEM: A guide for users and developers", J. Comput. Chem., 34, 1060-1070 (2013).

73 S. Y. S. Tan and E. I. Izgorodina, "Comparison of the effective fragment potential method with symmetry-adapted perturbation theory in the calculation of intermolecular energies for ionic liquids", J. Chem. Theory Comput., 12, 2553-2568 (2016).

74 J. Řezác, K. E. Riley, and P. Hobza, "S66: A well-balanced database of benchmark interaction energies relevant to biomolecular structures", J. Chem. Theory Comput., 7, 2427-2438 (2011). Erratum: J. Chem. Theory Comput. 10, 1359-1360 (2014).

75 "Ohio $\quad$ Supercomputer $\quad$ Center", 